Ces dernières décennies, de nombreux pays de l'OCDE ont augmenté de façon significative la proportion d'enfants préscolarisés.

Depuis quelques décennies, de nombreux pays ont augmenté de façon significative la proportion de jeunes enfants préscolarisés. Dans la majorité des pays de I'OCDE, la plupart des enfants sont scolarisés bien avant l'âge de 5 ans, et dans tous les pays de I'OCDE, plus des trois quarts des enfants de 4 ans (79\%) sont inscrits dans des programmes d'éducation ou d'accueil des jeunes enfants. Cependant, dans les pays de l'OCDE, les taux de participation varient de plus de 95 \% en Allemagne, en Belgique, en Espagne, en France, en Islande, en Italie, au Japon, au Luxembourg, au Mexique, en Nouvelle-Zélande, en Norvège, aux Pays-Bas et au Royaume-Uni, dans la partie haute de la fourchette, à moins de $60 \%$ (mais plus de $30 \%$ ) en Australie, au Canada, en Finlande, en Grèce, en Pologne et en Suisse, et jusqu'à moins de $30 \%$ en Turquie dans la partie basse de la fourchette.

Au Mexique et en Pologne, les taux de participation ont augmenté de plus de 20 points de pourcentage entre 2005 et 2010 (voir le graphique ci-dessous).

Taux de préscolarisation à l'âge de 4 ans

dans l'enseignement préprimaire et primaire (2005 et 2010)

Effectifs préscolarisés à temps plein et à temps partiel dans des structures publiques et privées d'accueil de la petite enfance

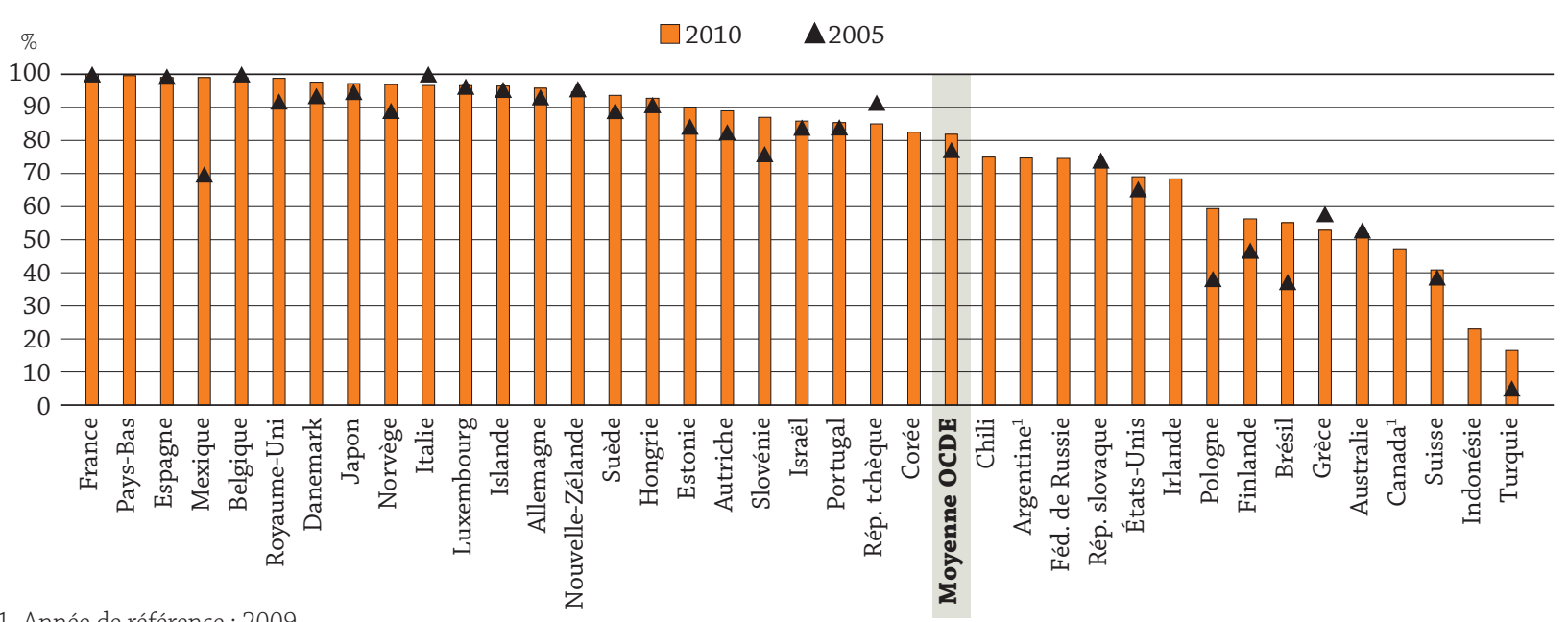

1. Année de référence : 2009.

Les pays sont classés par ordre décroissant du taux de préscolarisation à l'âge de 4 ans en 2010.

Source : OCDE. Données relatives à l'Argentine et l'Indonésie : Institut de statistique de l'UNESCO (Programme des indicateurs de l'éducation dans le monde). Regards sur l'éducation 2012 : Les indicateurs de l'OCDE, indicateur C2 (http://www.oecd.org/fr/edu/rse2012.htm).

Les résultats de l'enquête PISA montrent qu'une préscolarisation de qualité peut favoriser de meilleurs résultats plus tard dans la vie. Cependant, l'ampleur des bienfaits liés à la préscolarisation dépend fortement de la qualité des services offerts.

Un corpus de recherches de plus en plus important montre que I'EAJE aide à améliorer les capacités cognitives des enfants ainsi que leur développement socio-émotionnel, à jeter les bases de l'apprentissage tout au long de la vie, à rendre les résultats d'apprentissage des élèves plus équitables, à réduire la pauvreté et à améliorer la mobilité sociale d'une génération à l'autre (OCDE, 2012a; 2012b). Toutefois, la mesure dans laquelle l'EAJE peut générer ces bienfaits dépend de la qualité des services proposés. Accroître l'accès à ces services sans se préoccuper suffisamment de leur qualité ne permettra pas d'obtenir de bons résultats pour les élèves ou d'engendrer une amélioration de la productivité pour la société.

Les résultats de l'enquête PISA montrent que la préscolarisation est fortement associée à la performance en compréhension de l'écrit à l'âge de 15 ans, même après contrôle du milieu socio-économique des élèves. L'enquête PISA suggère également que la relation entre la préscolarisation et les résultats d'apprentissage ultérieurs est la plus forte dans les pays qui font état d'un certain niveau de qualité des services d'EAJE. Les indicateurs de qualité comprennent notamment le taux d'encadrement des enfants, la durée des programmes et le montant des dépenses publiques par enfant (OCDE, 2010). 


\section{INDICATEURS DE L'ÉDUCATION À LA LOUPE}

Les indicateurs liés aux environnements propices à l'apprentissage et au bien-être, tels que le taux d'encadrement des enfants et la qualité du personnel, ont un impact sur les résultats d'apprentissage des élèves.

En l'absence de mesures directes de la qualité des services, le taux d'encadrement des enfants est souvent utilisé comme indicateur de la qualité de l'éducation des jeunes enfants (OCDE, 2010). De nombreuses recherches ont montré que plus le taux d'encadrement est élevé, meilleurs sont les résultats des élèves aux évaluations cognitives (mathématiques et sciences) et linguistiques (langue d'instruction, lecture et reconnaissance des mots).

En moyenne, au niveau préscolaire, on compte 14 enfants pour chaque enseignant dans les pays de l'OCDE. Le rapport élèves-enseignant (auxiliaires d'éducation non inclus) varie de plus de 20 enfants par enseignant en France, en Israël, au Mexique et en Turquie, à moins de 10 au Chili, en Islande, en Nouvelle-Zélande, en Slovénie et en Suède (voir le graphique ci-dessous). II est à noter que certains pays utilisent beaucoup d'auxiliaires d'éducation au niveau préscolaire.

Les recherches démontrent que des praticiens qualifiés et une pédagogie de qualité sont à même de créer des environnements stimulants et enrichissants, et que les approches pédagogiques de meilleure qualité facilitent l'obtention de meilleurs résultats d'apprentissage (OCDE, 2012a). Les diplômes obtenus constituent l'une des variables prédictives les plus puissantes de la qualité du personnel. Il convient de noter toutefois qu'il ne s'agit pas tant du niveau de qualification en soi, mais plutôt du volume de formation spécialisée et pratique compris dans la formation initiale du personnel, du type de développement et formation professionnels proposé et suivi par les praticiens, et du nombre d'années d'expérience.

Il faut également noter que les conditions d'exercice de leur métier peuvent influencer le degré de satisfaction des professionnels sur leur lieu de travail, ce qui à terme affecte leur capacité et leur volonté de maintenir des relations stables, d'interagir et d'accorder suffisamment d'attention aux enfants (OCDE, 2012a). Une rotation trop élevée du personnel nuit à la continuité des soins, entame les efforts de développement professionnel, affecte la qualité globale et exerce un impact négatif sur les résultats des enfants.

Taux d'encadrement dans l'enseignement préprimaire (2010) Établissements publics et privés

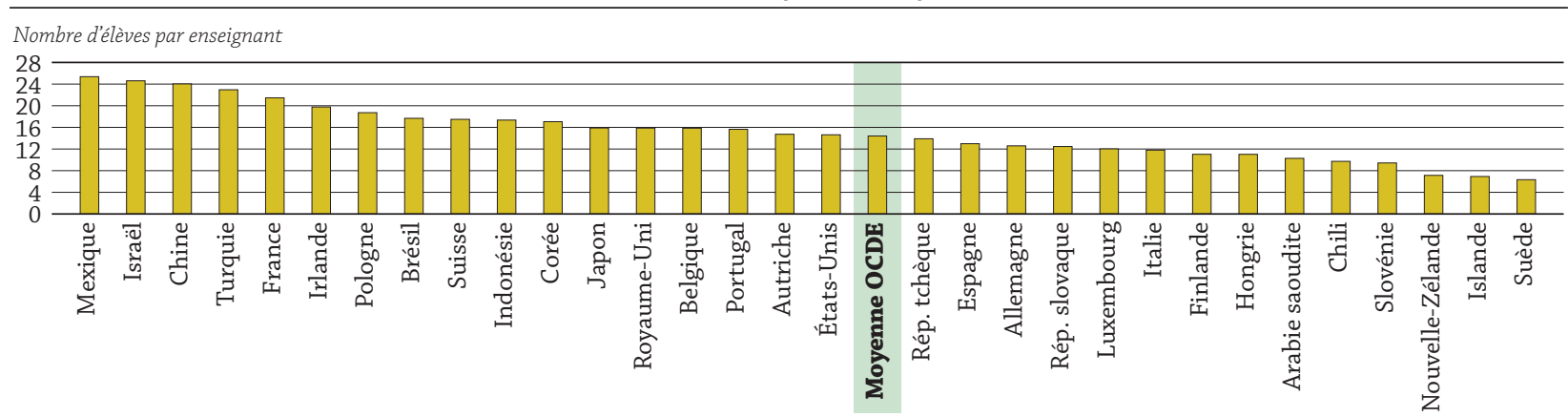

Les pays sont classés par ordre décroissant du taux d'encadrement dans l'enseignement préprimaire.

Source : OCDE. Données relatives à la Chine et l'Indonésie : Institut de statistique de l'UNESCO (Programme des indicateurs de l'éducation dans le monde). Données relatives à l'Arabie saoudite : Institut de statistique de l'UNESCO. Regards sur l'éducation 2012 : Les indicateurs de l'OCDE, indicateur C2 (http://www.oecd.org/fr/edu/rse2012.htm).

\section{La durée de la préscolarisation des jeunes enfants compte elle aussi. Dans la majorité des pays de l'OCDE, au moins une année de préscolarisation est proposée à titre gratuit.}

La durée de la préscolarisation joue également un rôle essentiel dans l'amélioration des résultats d'apprentissage plus tard dans la vie (OCDE, 2010). Afin d'assurer une certaine durée de participation aux programmes d'éducation des jeunes enfants, les pays créent souvent des droits pour garantir l'accès à une EAJE abordable et de qualité. De nombreux pays ont commencé à proposer des services « gratuits » d'éducation ou d'accueil des jeunes enfants à certains groupes d'âges, généralement une ou deux années avant le début de la scolarité obligatoire. Par exemple, les Pays-Bas offrent une préscolarisation gratuite aux enfants de 4 et 5 ans, tandis que celle-ci est proposée gratuitement pour les 3-4 ans en Angleterre et en Écosse. Certains pays ont étendu ce droit pour inclure des enfants encore plus jeunes : la France, Israël, le Mexique, le Portugal et la Suède offrent des services gratuits d'éducation ou d'accueil pour tous les enfants de 3 à 6 ans.

Pour donner accès plus tôt aux services d'éducation aux jeunes enfants, les pouvoirs publics peuvent également décider d'abaisser l'âge du début de la scolarité obligatoire. Du point de vue de l'équité, il s'agit là d'une option souvent considérée comme efficace car les inégalités sont susceptibles d'exister avant le début de la scolarité et ont tendance à se creuser lorsque celle-ci n'est pas obligatoire. 
Le niveau de financement public de l'EAJE joue également un rôle important. En l'absence d'un niveau suffisant de financement public, il y a davantage à craindre que l'accès aux programmes d'EAJE se limite aux seules familles aisées et que la qualité des programmes fluctue.

Les dépenses affectées à l'éducation des jeunes enfants représentent $9 \%$ des dépenses de l'OCDE au titre des établissements d'enseignement soit, en moyenne, $0.5 \%$ du PIB total. Les différences entre les pays sont significatives : par exemple, tandis que $0.1 \%$ du PIB, voire moins, est alloué à l'éducation des jeunes enfants en Australie et en Irlande, cette proportion atteint $0.8 \%$ ou plus au Danemark, en Espagne, en Islande et en Israël, parmi les pays de l'OCDE (voir le graphique ci-dessous).

Le financement public de l'éducation des jeunes enfants est le modèle qui prévaut dans les pays européens membres de l'OCDE par rapport aux pays de l'OCDE non européens. La part du financement privé varie fortement d'un pays à I'autre, de $5 \%$, voire davantage, en Allemagne et Autriche, à plus de $48 \%$ en Australie, en Corée et au Japon, parmi les pays de I'OCDE. Dans les pays qui ne parviennent pas à affecter suffisamment de fonds publics pour assurer la quantité et la qualité des services d'EAJE, il est probable que certains parents soient plus enclins à inscrire leurs enfants dans des structures privées, ce qui peut s'avérer très coûteux (OCDE, 2012b), tandis que d'autres préféreront rester à la maison avec leur enfant, ce qui a pour effet de limiter la participation des femmes à la population active (OCDE, 2011).

Dépenses au titre des établissements d'enseignement préprimaire, en pourcentage du PIB (2009) Selon la provenance des fonds

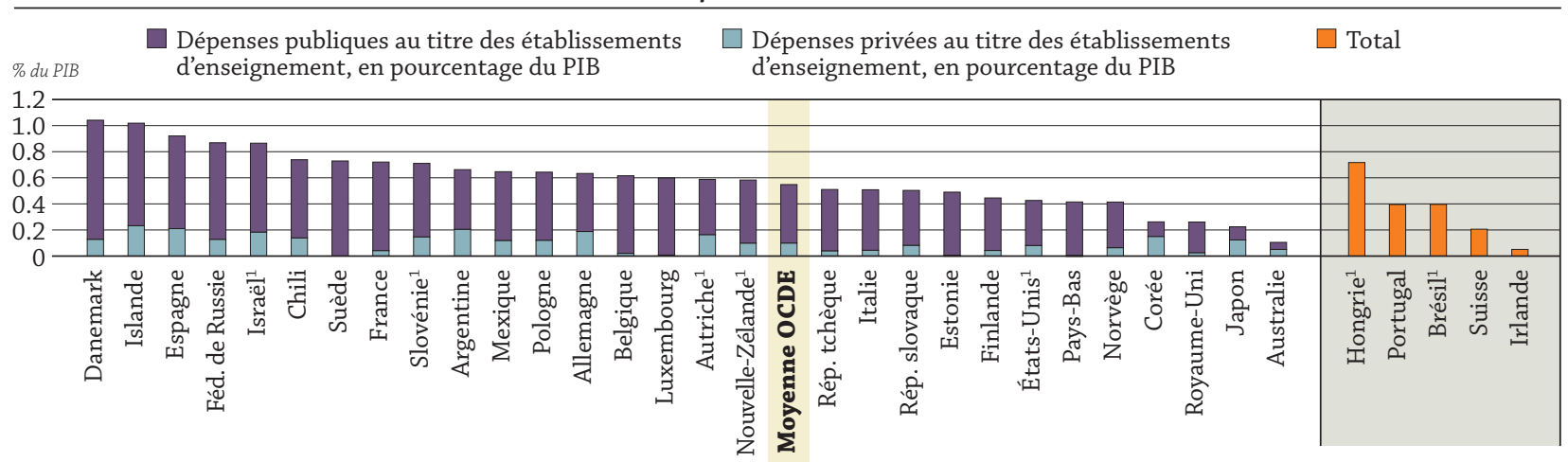

1. Certaines dépenses au titre des services de garde de la petite enfance sont incluses.

Les pays sont classés par ordre décroissant des dépenses publiques et privées au titre des établissements d'enseignement.

Source : OCDE. Données relatives à l'Argentine: Institut de statistique de l'UNESCO (Programme des indicateurs de l'éducation dans le monde). Regards sur l'éducation 2012 : Les indicateurs de l'OCDE, indicateur C2 (http://www.oecd.org/fr/edu/rse2012.htm).

\section{Références}

OCDE (2006), Petite enfance, grands défis II : Éducation et structures d'accueil, Éditions OCDE, Paris.

OCDE (2010), Résultats du PISA 2009 : Surmonter le milieu social (volume II), PISA, Éditions OCDE, Paris.

OCDE (2011), Assurer le bien-être des familles, Éditions OCDE, Paris.

OCDE (2012a), Petite enfance, grands défis III : Boîte à outils pour une éducation et des structures d'accueil de qualité, Éditions OCDE, Paris. OCDE (2012b), Regards sur l'éducation 2012 : Les indicateurs de l'OCDE, Éditions OCDE, Paris.

Pour conclure Dans des temps de consolidation budgétaire, assurer l'accès à des services d'EAJE de qualité devrait rester une priorité pour améliorer les résultats d'apprentissage des enfants et engendrer des gains d'efficacité à long terme pour la société dans son ensemble, tout en protégeant les plus démunis.

Voir :

www.oecd.org/edu/ earlychildhood/toolbox

www.oecd.org/edul earlychildhood

www.oecd.org/fr/els/ famille/
Consulter :

OCDE (2012a), Petite enfance, grands défis III: Boîte à outils pour une éducation et des structures d'accueil de qualité, Éditions OCDE, Paris.

OCDE (2012b), Regards sur l'éducation 2012: Les indicateurs de l'OCDE, Éditions OCDE, Paris.
Pour plus d'informations, contacter :

Miho Taguma (Miho.Taguma@oecd.org)
Prochain numéro : Quels facteurs influent sur le niveau des dépenses d'éducation au titre du personnel enseignant? 